\title{
Atrial Infarction is a Unique and Often Unrecognized Clinical Entity
}

\author{
Rosana G. G. Mendes, Paulo Roberto B. Evora
}

Ribeirão Preto, SP - Brazil

\begin{abstract}
A patient with heart failure and acute atrial fibrillation received the final diagnosis of atrial infarction associated with ventricular infarction based on clinical findings of ischemia in association with atrial fibrillation and heart failure (mechanisms probably involved: contractile dysfunction and loss of atrial contribution). Although a transesophageal echocardiography, which could refine the diagnosis of anatomic abnormalities, was not performed, all evidence led to the diagnosis of atrial involvement. Electrocardiographic findings were consistent with Liu's major criterion 3. Therapy with digitalis, quinidine and angiotensin-converting enzyme inhibitors was chosen, as the patient had acute pulmonary edema. The use of beta-blockers and verapamil was res-tricted. No other complications, such as thrombo-embolism or atrial rupture, were noted.
\end{abstract}

So far, atrial infarction is a poorly studied condition. This fact is reflected by the lack of published reports in the literature. In contrast with ventricular infarction, it represents a unique clinical entity that, when diagnosed, requires significant changes in therapy. In this report, a patient presenting with heart failure and acute atrial fibrillation, who received a final diagnosis of atrial infarction associated with ventricular infarction, is described.

\section{Case report}

A 67-year-old man was hospitalized due to pneumonia. One week later, he developed dyspnea at rest and tachycardia. Ten days previously, he had been treated for a dyspeptic disease due to severe epigastric pain. His physical examination showed marked bilateral pulmonary rales on auscultation, extending to the medium third of his lungs. He had an irregular heart rate and an apical systolic murmur $++/ 6+$. Inferior limb edema, as well as varicose veins, were not noted.

The results of the diagnostic workup included: 1) chest X-ray: an enlarged left ventricle (LV) and a bilateral alveolar infiltrate in pulmonary bases; 2) electrocardiogram
(ECG): atrial fibrillation, necrosis and ST segment elevation in the lateral wall (fig. 1); 3) Transthoracic echocardiogram: lateral hypokinesis, papillary muscle dysfunction with a slight mitral regur-gitation; 4) Routine laboratory test results within the normal range, with slightly increased cardiac enzymes.

After treatment for pulmonary edema and reversion of the atrial fibrillation (with the use of digitalis, diuretics, angiotensin-converting enzyme inhibitors, oxygen and quinidine), the clinical condition was controlled and the sinus rhythm was restored. A new control ECG showed sinus rhythm, $\mathrm{Q}$ waves and ST segment elevation in the lateral wall, PR-segment depression in leads DI, DII, $\mathrm{V}_{4}, \mathrm{~V}_{5} \mathrm{e}$ $\mathrm{V}_{6}$, as well as clear PR-segment elevation in leads $\mathrm{V}_{1}$ and $\mathrm{V}_{2}$ (figs. 2 and 3). At this moment, the diagnosis of atrial infarction associated with myocardial infarction was considered in view of the ischemic abnor-malities associated with the atrial arrhythmia.

During clinical investigation, cardiac catheterization with coronary angiography showed: a $30 \%$ obstruction of the dominant right coronary artery in its medium third, a normal left main coronary artery, a $90 \%$ obstruction of the anterior descending coronary artery next to the first septal branch, a 70\% obstruction of the circumflex artery at the origin of posterior ventricular and left marginal branches, a subocclusion in the emergence of the ramus medianus, apical akinesia and a slight mitral regurgitation.

As the patient was clinically stable, he underwent a coronary artery bypass graft surgery with in situ anastomosis of the left mammary artery to the anterior descending coronary artery and saphenous vein bypass grafting to the ramus medianus and circumflex artery. In the days following surgery, he developed pleuropericarditis that improved only after the use of corticosteroids and required a few pleural drainages due to fluid accumulation.

The last transthoracic echocardiogram performed showed normally functioning $\mathrm{LV}$, slight dilation of the left atrium (LA), slight mitral regurgitation and no pericardial effusion.

\section{Discussion}

Most atrial infarctions occur as a result of atherosclerotic heart disease. In addition to atherosclerotic 


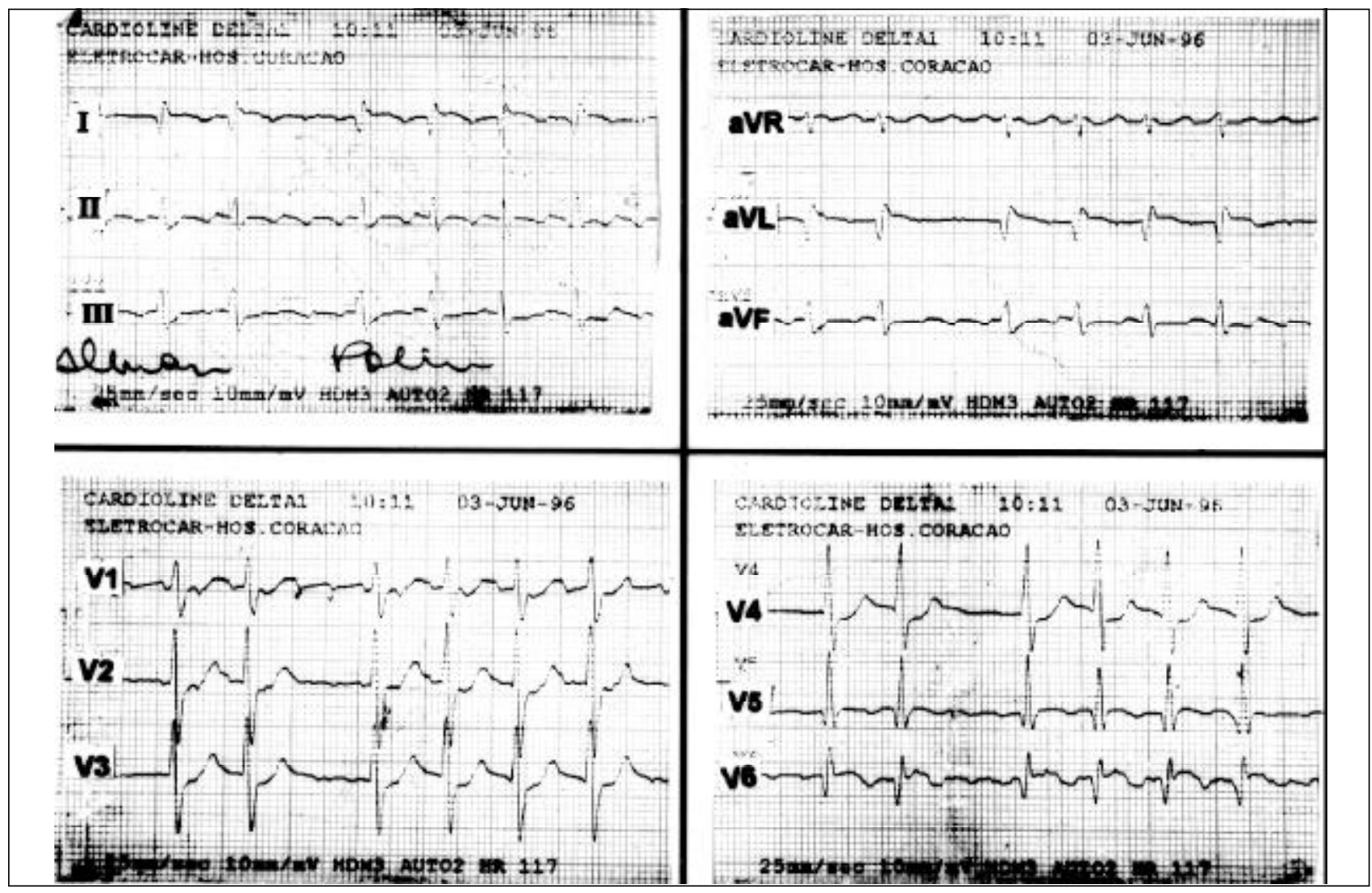

Fig. 1 - Initial electrocardiogram showing atrial fibrillation, necrosis and subepicardial current of injury in the lateral and upper lateral areas.

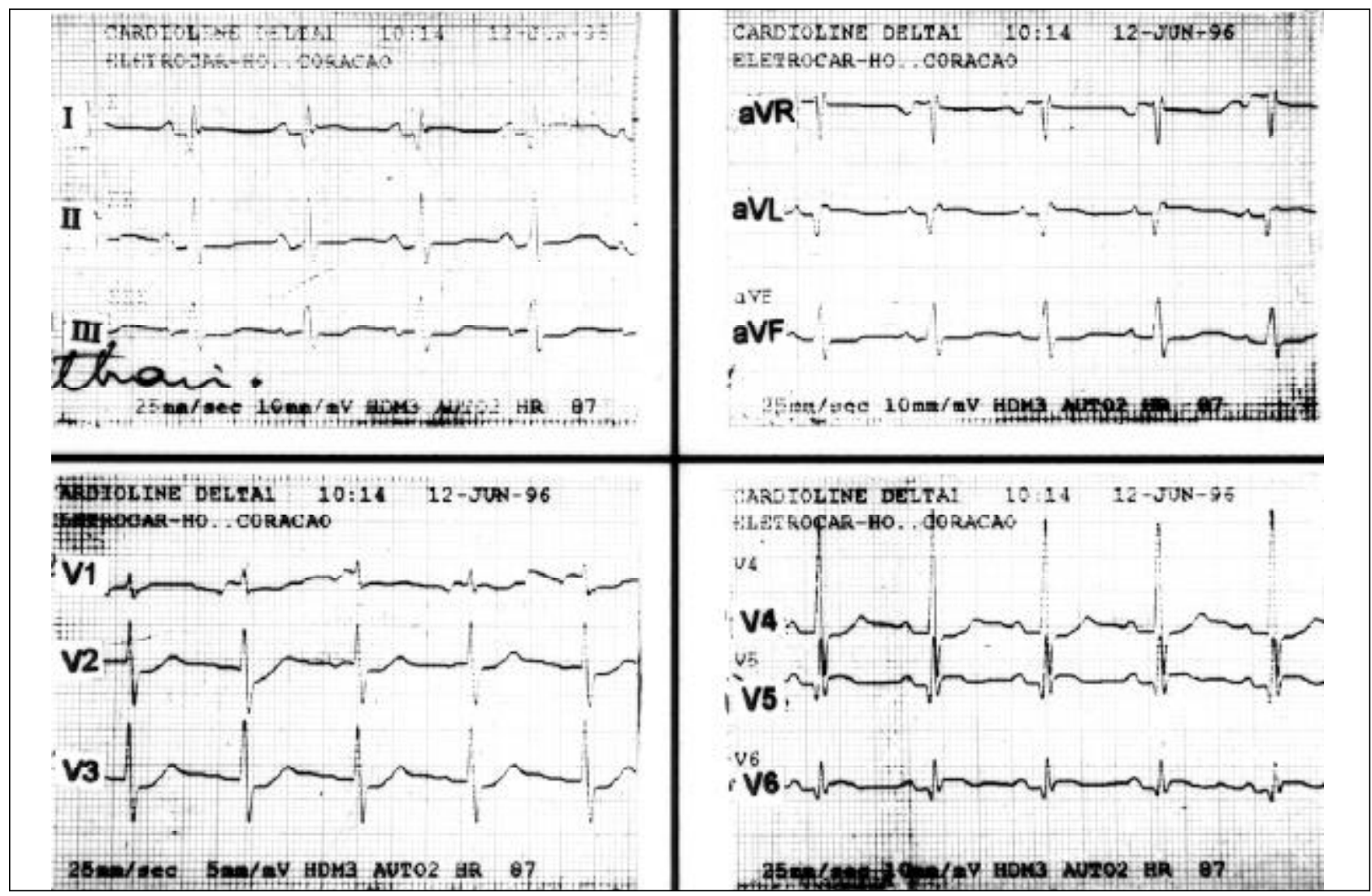

Fig. 2 - Patient's electrocardiogram showing PR segment abnormalities consistent with the diagnosis of atrial infarction: control ECG showed sinus rhythm, with necrosis and ST segment elevation in the lateral wall, PR-segment depression in leads DI, DII, $\mathrm{V}_{4}, \mathrm{~V}_{5}$ and $\mathrm{V}_{6}$, as well as clear PR-segment elevation in leads $\mathrm{V}_{1}$ and $\mathrm{V}_{2}$. 


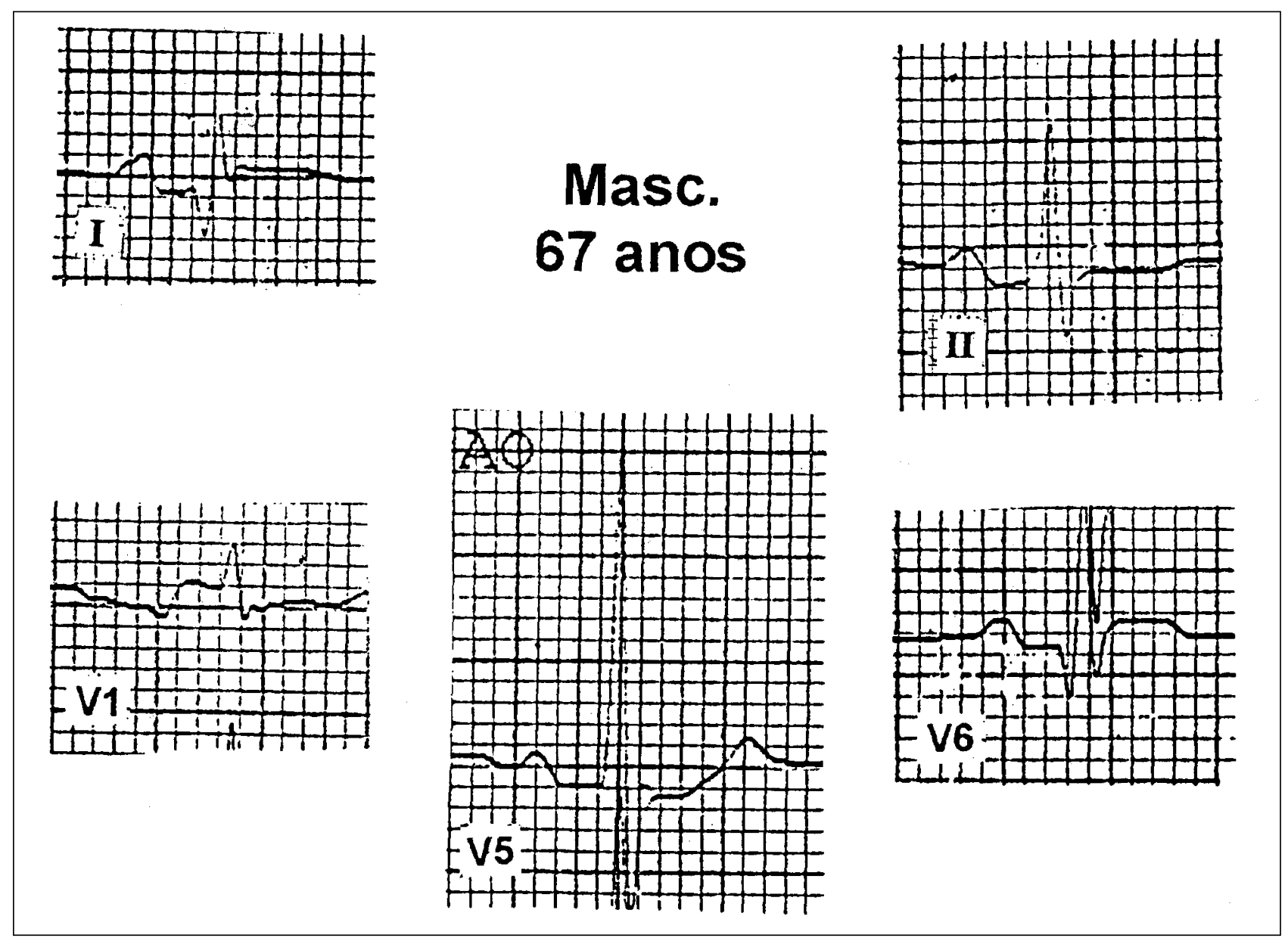

Fig. 3 - Amplification of leads showing in detail PR-segment abnormalities consistent with the diagnosis of atrial infarction in leads DI, DII, $\mathrm{V}_{1}, \mathrm{~V}_{5}$ and $\mathrm{V}_{6}$.

disease, atrial infarctions have been less often related to: 1 ) chronic obstructive pulmonary disease with cor pulmonale and normal coronary arteries; 2 ) primary pulmonary hypertension; 3) muscular dystrophy;4) Friedreich's ataxia and 5) aluminum phosphide intoxication. A comprehensive report was published by Lazar et $\mathrm{al}^{1}$, with an excellent review of the history, incidence, anatomy, pathology and etiological, clinical and diagnostic aspects of acute atrial infarction.

This case report was motivated by the following aspects of atrial infarction, based on the selected bibliographic references: 1) so far, atrial infarction has been a poorly studied condition. This fact is reflected by the scarcity of published reports in the literature; 2) atrial infarction represents a unique clinical entity apart from ventricular infarction and, when diagnosed, requires significant changes in therapy; 3) in routine medical practice, it is felt that the diagnosis of atrial infarction has little clinical relevance; 4) the prognosis of atrial infarction, more than its occurrence per se, is related to the associated ventricular infarction; 5) in addition to the associated supraventricular arrhythmias, atrial infarction may present with lethal complications, such as thromboembolism and atrial rupture; 6) in order to make the diagnosis, with the patient alive or postmortem, the physician must be aware of this condition and pay atten- tion to the possible electrocardiographic abnormalities of the PR-segment and to the higher incidence of supraventricular arrhythmias compared with ventricular infarctions and 7) diagnosis in daily routine practice is based on electrocardiographic findings and on the transthoracic echocardiogram. However, these simpler tests are limited. More appropriate imaging techniques, such as transesophageal echocardiography, are required, as well as the acquisition of new diagnostic resources, that is, the use of graphic resources with increased resolution.

In most cases, atrial infarction involves the right side of the heart. Its characteristics may be divided into two groups: 1) those cases where the effects of ventricular infarction predominate and 2) those that occur as a direct result of atrial involvement. Many times it is difficult to make this distinction. In addition, it is of note that a series of complications resulting from atrial infarction may occur, including arrhythmias, rupture, thromboembolic phenomena and reduced heart output as a result of the loss of atrial contribution ${ }^{1,2}$.

Standard ECG has been the primary diagnostic tool when the patient is alive, and it deserves to be discussed in more detail. Due to the low voltage generated during this test and the reduced thickness of the atrial wall, atrial infarc- 
tion abnormalities may often be absent or hidden by increased ventricular depolarization ${ }^{1,3}$. However, some electrical criteria for the diagnosis of atrial infarction have been proposed and include: abnormalities of the PTa-segment and $\mathrm{P}$ wave morphology (fig. 4), as well as the development of supraventricular arrhythmias ${ }^{3}$. A large review of a series of reports concludes that there are no specific data for the electrocardiographic diagnosis of atrial infarction ${ }^{4}$. The most commonly used electrocardiographic diagnostic criteria have been those proposed by Liu et al, who categorized them into major and minor criteria (Table I) ${ }^{5}$. More recently, Mayuga \& Singer $^{6}$ suggested the addition of the following electrocardiographic criteria to those established by Liu: 1) follow-up abnormalities of $\mathrm{P}$ wave shape, J-point, and PR-segment in serial ECG tracings and 2) simultaneous occurrence of atrial arrhythmias and abnormalities of $\mathrm{P}$ wave shape and PR-segment. These authors emphasize that preexistent $P$ wave abnormalities must be taken into consideration when interpreting Liu's criteria. Maybe the use of specialized electrical tracings with high resolution (atriograms or transesophageal recordings), as well as intraatrial tracings during cardiac catheterization, may be more specific for the diagnosis of atrial infarction.

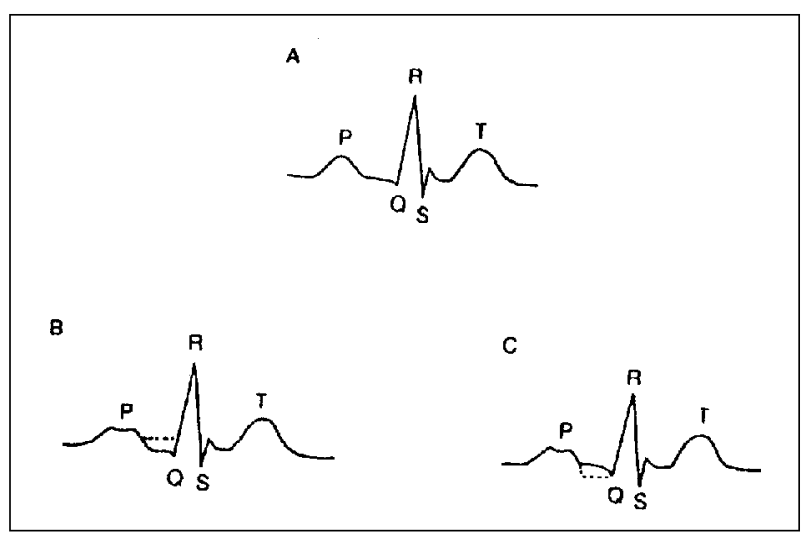

Fig. 4 - P wave morphology and PR-segment abnormalities in atrial infarction. A) Normal $\mathrm{P}$ wave and PR-segment; $\mathrm{B}$ and $\mathrm{C}$ ) $\mathrm{P}$ wave abnormalities and elevation and depression of the PR-segment, respectively (Adapted ${ }^{7}$ ).

Some reports mention the diagnosis of atrial infarction with the use of radioisotopes, as well as the echocardiographic diagnosis of this condition. The transthoracic echocardiography is limited by the difficulty in visualizing the atria, especially the right atrium. However, transesophageal echocardiography allows a better visualization
Table I - Electrocardiographic diagnostic criteria according to Liu et $\mathbf{a l}^{5}$.

Major criteria:

1) PTa-segment elevation $>0.5 \mathrm{~mm}$ in leads $V_{3}$ and $V_{6}$ with reciprocal depression of PTa segments in $\mathrm{V}_{1}$ and $\mathrm{V}_{2}$ leads.

2) PTa-segment elevation $>0.5 \mathrm{~mm}$ in lead I with reciprocal depressions in leads II and III.

3) PTa-segment depression $>1.5 \mathrm{~mm}$ in precordial leads and $1.2 \mathrm{~mm}$ in leads I, II and III, associated with any atrial arrhythmia.

Minor criteria:

Abormal P-waves, flattening of $\mathrm{P}$-wave in $\mathrm{M}$, flattening of $\mathrm{P}$-wave in $\mathrm{W}$, irregular or notched $\mathrm{P}$ wave.

of cardiac atrial chambers and may be an obligatory test to confirm the presence of atrial infarction and thrombi ${ }^{7}$.

Some aspects deserve special mention in the routine management of acute myocardial infarction (AMI): 1) the treatment of supraventricular arrhythmias; 2) the use of anticoagulation due to the frequency of thromboembolism and 3 ) the treatment of heart failure, when this condition is present. The use of beta-blockers requires special attention. This group of drugs has been proved useful in decreasing the frequency of tachyarrhythmias and atrial fibrillation/ flutter during hospitalization. It is not clear, however, whether this effect occurs as a result of an antiarrhythmic effect or due to the decrease in the size of the infarction. One can assume, although without a definite conclusion, that patients with atrial infarction may benefit from the early use of beta-blockers. Atrial infarction alone does not seem to cause heart failure. Thus, poor responses with the use of beta-blockers are not expected ${ }^{1}$.

In conclusion, this case report has some aspects that deserve special mention: 1) the presence of ischemia associated with atrial fibrillation and heart failure (mecha-nisms probably involved: contractile dysfunction and loss of atrial contribution) which led to the diagnosis of atrial infarction associated with AMI; 2) transesophageal echocardiography was not performed; this test could have refined the diagnosis of anatomic abnormalities, but all evidence led to the diagnosis of atrial involvement; 3 ) electrocardiographic findings were consistent with Liu's major criterion 3;4) the therapy, which included digitalis, quinidine and angiotensin-converting enzyme inhibitors, was chosen because the patient had acute pulmonary edema and the use of betablockers and verapamil was restricted; 5) the patient did not have other complications such as thromboembolism or atrial rupture. 


\section{References}

1. Lazar EJ, Goldberger J, Peled H, Sherman M, Frishman WH. Atrial infarction: diagnosis and management. Am Heart J 1988; 116: 1058-63.

2. Alonso-Orcajo N, Izquierdo-Garcia F, Simarro E. Atrial rupture and sudden death following atrial infarction. Int J Cardiol 1994; 46: 82-4.

3. Liu CK, Greenspan G, Piccirilio RT. Atrial infarction of the heart. Circulation 1961; 23: 331-8

4. Christensen JH, Nielsen FE, Falstie-Jensen N, Schmidt EB. Interobserver variation in interpretation of electrocardiographic signs of atrial infarction. Clin Cardiol 1993; 16: 603-6.
5. Mayuga RD, Singer DH. Atrial infarction: clinical significance and diagnostic criteria. Pratical Cardiol 1985; 11: 142-60.

6. Vargas-Barron J, Romero-Cardenas A, Keirns C, et al. Transesophageal echocardiography and right atrial infarction. J Am Soc Echocardiogr 1993; 6 : 543-7.

7. Nielsen FE, Andersen HH, Gram-Hansen P, Sorensen HT, Klausen IC. The relationship between ECG signs of atrial infarction and the development of supraventricular arrhythmias in patients with acute myocardial infarction. Am Heart J 1992; 123: 69-72. 